\title{
Rationality of Fixed Dose Combinations of Central Nervous System Drugs in a Tertiary Care Hospital
}

\author{
Anilasree B.P., Anuroopa K.P., Arun Jyothi S., Basila T.*, Swathi Saji \\ Department of Pharmacy Practice, Devaki Amma Memorial College of Pharmacy, Chelembra, Malappuram, Kerala
}

\begin{abstract}
Objectives: To analyze the rationality of central nervous system fixed dose combinations used in a tertiary care hospital. Methodology: The study was an hospital based observational study. The data was collected from an annual drug compendium entitled "Hospital Drug List". Fixed dose combinations (FDCs) enlisted in central nervous system (CNS) sections were selected for the study purpose. The active pharmacological ingredients (APIs) in FDC was checked for approval by Drug Control General of India (DCGI), World Health Organization (WHO) and essential medicine (EML)/national essential medicine list (NEML),both or none and all the ingredients (molecule, excipients) present in the FDC was checked whether banned or under any controversies in India as well as worldwide. Efficacy and safety of the individual active pharmacological ingredients (APIs) and their combination were searched. Details of each drug were collected [Generic name, Pharmacokinetics, Interaction affected, Pharmacodynamics, and Advantages of FDCs]. The data collected was analyzed by a tool to assess the rationality of fixed dose combinations which is pre-tested and validated by Shah et al., based on WHO guidelines. Result and Discussion: A total of 25 CNS FDCs were taken, on assessment of CNS FDCs 21 (84\%) were found to be rational and 4 (16\%) were found to be irrational with the mean rationality score of 7.2. By winding up, state of nonbeing, absenteeism of legality and effectiveness of the formulations appeared in to a peculiar combinations and inadequate practice. The approval process of these combinations by various committees should be robust.
\end{abstract}

Keywords: Rationality; Fixed Dose Combinations; CNS Drugs; Safety and Efficacy.

Article Info: Received 10 July 2019; Review Completed 13 August 2019; Accepted 18 August 2019; Available online 30 Aug 2019

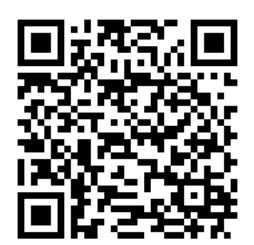

Cite this article as:

Anilasree BP, Anuroopa KP, Arun Jyothi S, Basila T, Swathi S, Rationality of Fixed Dose Combinations of Central Nervous System Drugs in a Tertiary Care Hospital, Journal of Drug Delivery and Therapeutics. 2019; 9(4-A):15-18 http://dx.doi.org/10.22270/jddt.v9i4-A.3387

Basila.T, Department of Pharmacy Practice, Devaki Amma Memorial College of Pharmacy, Chelembra, Malappuram, Kerala

\section{INTRODUCTION}

Fixed Dose combinations (FDCs) are defined by the World Health Organization (WHO) as combination of two or more active ingredients in a fixed ratio of dose. ${ }^{1}$ FDCs must be shown to be safe and effective for the claimed indications and it cannot be assumed that benefits of the FDCs outweigh its risks. ${ }^{2}$ In last six years Central Drugs Standard Control Organization (CDSCO) has approved 303 FDCs. 3 This number is large when compared to the Essential Medicines List (EML) of WHO and national list of essential medicine (NEML). 4, 5 As of now studies were mainly focusing on prescribing pattern of FDCs and their rationality. 6 Rationality status of many fixed dose combinations marketed in India is not clear. The rationality of FDCs is based on certain aspects which are as follows, the drugs in the combination should act by different mechanisms, pharmacokinetic must not be widely different and combination should not have supra-additive toxicity of the ingredients.
CNS drugs are the medications which are used to treat various neurological and psychiatric conditions. Drugs that act in the CNS are invaluable therapeutically. They can, e.g., relieve pain, reduce fever, suppress disorder movements, induce sleep or arousal, reduce appetite, and suppress nausea. Selectively acting drugs can be used to treat anxiety, depression, mania or schizophrenia and do so without altering consciousness. However, the excessive use of such drugs can affect lives adversely when uncontrolled, and can cause toxic side effects ${ }^{7}$. It is clear that nearly all drugs with CNS effects act on specific receptors that modulate synaptic transmission. While a few agents such as general anesthetics and alcohol may have nonspecific actions on membranes, even these non-receptor-mediated actions result in demonstrable alterations in synaptic transmission. 8 The CNS is responsible for processing and controlling most of our bodily functions, and consists of the nerves in the brain and spinal cord. There are many different types of drugs that work on the CNS, including anesthetics, anticonvulsants, antiemetic, antiparkinsons agents, CNS stimulants, muscle relaxants, narcotic analgesics, nonnarcotic analgesics and 
sedatives. Hence there is a need to study pattern of prescription of these fixed dose combination drugs and know the rationality behind there usage.

\section{MATERIALS AND METHODS:}

\section{Ethics:}

Ethical clearance was obtained from Institutional Review Board, Devaki Amma Memorial College of Pharmacy, Chelembra, Malappuram, Kerala and approved by the Institutional Ethics Committee, P. V. S. Hospital (P) LTD, Calicut, Kerala (Ref. No. PVS/EC/02/17-18).

\section{Study design:}

A hospital based observational study conducted from November 2017 to April 2018 on 25 Central nervous system (CNS) fixed dose combinations (FDCs) which were taken from PVS hospital drug compendium.

\section{Study Materials:}

- Hospital drug list from PVS Hospital (P) LTD, Calicut.

- Standard reference books of pharmacology and medicine.

- Authentic data base like PubMed, Medline, Google scholar and Cochrane data.

- Literatures relevant to the study.

- $\quad$ List of fixed dose combinations (FDCs) published by World Health Organization (WHO) and Drug Controller General of India (DCGI).

\section{Study Procedure:}

For the conduct of study hospital pharmacy of the study site was approached and the lists of the FDCs were collected from an annual compendium entitled "Hospital Drug List".

FDCs enlisted in central nervous system sections were collected and by Using Shah et al., pre tested validated tool based on WHO guidelines certain parameters were evaluated.

The active pharmacological ingredient (APIs) in FDC was checked for approval by DCGI. If it is approved then scored as $(+1)$ and if not $(-1)$.

All the ingredients (molecule, excipients) present in the FDC was determined and checked whether banned or under any controversies in India as well as worldwide.

Drugs taken from the hospital list were checked whether they are present in WHO, essential medicine list $(E M L) /$ national essential medicine list (NEML), both or none.

If the drug was present in both the EMLs or any one of the EML, score given was (+1) and if not present in any then scored as (0).

Efficacy and safety information of the APIs and FDCs regarding these drugs was collected from the textbooks, reference books and authenticated websites.

If the APIs or FDCs was safe and effective the score was (+1) and if not (0).

Pharmacokinetic parameters were obtained from text books etc. and these parameters were used to check for interaction. If the interaction was favorable score was $(+1)$, unfavorable $(-1)$ and not affected score was (0).

The pharmacodynamic interaction was reviewed and mechanism of action of each ingredient is checked. If similar mechanism of action score was (0), if different mechanism of action score was (+1).

Advantages of FDCs were checked,

a) If FDCs is more beneficial than the individual compound to reduce the disease then the score was $(+1)$.

b) If FDCs has lesser adverse drug reactions the score was $(+1)$.

c) If FDCs used was more convenient than individual compounds then the score was $(+1)$.

Total score obtained after assessing these parameters are added up,

a) If the score was greater than or equal to seven, FDCs was considered as rational.

b) If the score was less than or equal to six, FDCs was considered as irrational.

\section{RESULTS:}

The data collected was analyzed by a tool to assess the rationality of fixed dose combinations which is pre-tested and validated by Shah et al., ${ }^{9}$ based on WHO guidelines. Based on the analysis the results are given in the following.

\section{Assessment of Rationality}

A total of 25 central nervous system fixed dose combinations were selected.

\section{Details of Active Pharmacological Ingredients}

Out of 25 CNS FDCs, 15 (60\%) FDCs contained API approved by DCGI, in which 13 (52\%) were rational and 2 (8\%) were irrational. Remaining $10(40 \%)$ FDCs had one or more unapproved API, in which 8 (32\%) were rational and 2 (8\%) were irrational.

Table 1: Rationality of APIs in the FDC list approved by DCGI

\begin{tabular}{|l|l|}
\hline Parameters & $\begin{array}{l}\text { No of CNS FDCs } \\
\text { (n=25) }\end{array}$ \\
\hline APIs approved by DCGI + Rational & $13(52 \%)$ \\
\hline APIs approved by DCGI + Irrational & $2(8 \%)$ \\
\hline APIs unapproved by DCGI + Rational & $8(32 \%)$ \\
\hline APIs unapproved by DCGI + Irrational & $2(8 \%)$ \\
\hline
\end{tabular}

\section{FDCs containing Banned/Controversial ingredients}

Out of 25 CNS FDCs assessed, 3 (12\%) were found to be banned or controversial.

\section{Listing in Essential Medicine List (EML)}

Out of the 25 assessed CNS FDCs, 11 (44\%) combinations APIs were not present in both WHO/National EML, 7 (28\%) combinations APIs were present in either any one of the EML (WHO/National) and 7 (28\%) combinations APIs were present in both the EML.

\section{Evidence of Efficacy and Safety of API \& FDC}

Out of 25 CNS FDCs, APIs efficacy and safety of 16 (64\%) FDCs were well documented and proved in clinical trials or meta-analysis. 1 (4\%) FDCs had neither safe nor effective APIs.

Out of 25 CNS FDCs, FDCs efficacy and safety of 16 (64\%) were well documented and proved in clinical trials or metaanalysis. While $1(4 \%)$ FDCs were found to be safe but not 
effective, 5 (20\%) FDCs were found to be effective but didn't show safety. $3(12 \%)$ of the CNS FDCs was neither safe nor effective.

\section{Assessment of Pharmacokinetic and Pharmacodynamic interaction}

Favorable pharmacokinetic interaction was found in 5 (20\%) FDCs. No interaction between APIs was found in 20 (80\%) FDCs.

All 25 FDCs, API had different mechanism of action.

\section{Advantage(s) of FDC}

Out of 25 CNS FDCs, 6 (24\%) showed advantage of reducing Adverse Drug Reactions (ADRs) as compared to individual ingredient. To a great surprise, all FDCs showed advantage of being convenient by reducing pill count or frequency of administration. Also 6 (24\%) of the FDCs showed advantage of dose reduction of individual active ingredient.

All the above obtained data was analyzed. For the analysis the FDCs were categorized in to four groups.

Table 2: Rationality of FDCs approved by DCGI

\begin{tabular}{|c|c|c|}
\hline Groups & Description & No. of CNS drugs \\
\hline A & DCGI approved + Rational & $16(64 \%)$ \\
\hline B & DCGI approved + Irrational & $2(8 \%)$ \\
\hline C & DCGI approved + Rational & $5(20 \%)$ \\
\hline D & DCGI approved + Irrational & $2(8 \%)$ \\
\hline
\end{tabular}

On assessment of 25 CNS FDCs 21 (84\%) were found to be rational and $4(16 \%)$ were found to be irrational with the mean rationality score of 7.2

Table 3: Assessment of CNS FDCs using Rationality Tool $(n=55)$

\begin{tabular}{|c|c|}
\hline Parameters & CNS FDCs (n=25) \\
\hline DCGI Approved & 15 \\
\hline Mean rationality score & 7.2 \\
\hline Number of rational FDCs & 21 \\
\hline Number of irrational FDCs & 4 \\
\hline Number of API in each FDCs \\
\hline $\mathbf{2}$ & 23 \\
\hline $\mathbf{3}$ & 2 \\
\hline $\mathbf{4}$ & 0 \\
\hline
\end{tabular}

\section{DISCUSSION}

The present study showed that a substantial number of rational CNS FDCs were available in the annual drug compendium. All though the Drug regulators of India prevent the manufacture, distribution, sale of irrational FDCs, lack of coordination with state licensing authorities and system to critically analyze the scientific validity has resulted in mushrooming of few formulations present in the formulary. Popularity of FDCs is increasing rapidly in case if more than one disease is present in patients.

The studies showed that majority of these FDCs were rational and approved by DCGI. While APIs used in the majority of these formulations are individually approved by Central Regulatory authority, few FDCs contained unapproved APIs and found to be rational and few were irrational. Surprisingly, only 3 CNS FDCs (aceclofenac plus paracetamol, etodolac plus paracetamol, paracetamol plus dextropropoxyphene) were included in banned drug list which was present in the formulary of the Hospital. Availability of these FDCs raises safety concern and is important from a public health perspective.

Further, evidence of efficacy and safety of individual APIs was found, the scientific validity of majority of CNS FDCs was lacking. More trials need to be conducted as some were lacking published articles related to the evidence of safety and efficacy. Safety and efficacy is of at most importance when two drugs are combined together as a single formulation.

Similarly, certain rational CNS FDCs were combination of dopamine precursors etc. The combination of levodopa plus carbidopa increases efficacy due to pharmacokinetic advantage. The addition of carbidopa decreases the peripheral metabolism of levodopa, thereby, increasing its availability. ${ }^{10}$ All the combinations were having different mechanism of action, which is good for rationality. But in this study, all criteria were used together to establish rationality. 11

The rationality tool also found that DCGI approved FDCs contain both rational and irrational FDCs. It can be suggested that irrational formulations can be banned or subjected for further efficacy and safety data. Interestingly, the rationality tool also showed small number of FDCs which were DCGI unapproved but rational, for example, tranexamic acid plus mefenamic acid. Due to scientific validity these FDCs may be considered for approved by central authorities. In addition, the assessment of rationality revealed that the mean rationality score of CNS FDCs was 7.2 more in rational FDCs irrespective of approval by central regulator authority. Thus, this further validates the rationality tool and it can be used by prospective researchers and regulatory body.

The study findings confirm that few potentially harmful, unapproved and irrational FDCs are being used in the study site (Tertiary care hospital). Although the data was obtained from annual drug compendium and the information needed were obtained from standard text books and reference books of pharmacology and medicine, authentic data base like PubMed, Medline, Google scholar and Cochrane data and literatures relevant to the study. A possibility of lack of complete information cannot be ruled out. However, the data analyzed leads to some important conclusions.

\section{ACKNOWLEDGEMENT:}

Authors like to express their sincere thanks to Dr. Samidh Shah for his suggestion to make tool for rationality assessment of fixed dose combinations. Also like to thank M. 
Narayanan, Prof. V. Mohandas, Dr. G. Babu, Dr. Biju C.R and Dr. Siraj Sundaran.

\section{CONFLICTS OF INTEREST: Nil}

\section{REFERENCES}

1. WHO Expert Committee on Specifications for Pharmaceutical Preparations: thirty- ninth report. (WHO technical report series; 929) 2005.

2. Policy guidelines for approval of fixed dose combinations (FDCs) in India

(www.cdsco.nic.in/writereaddata/Report\%20of\%20Dr.C.K\%20Kok ate.pdf).

3. Fixed dose combinations approved by DCGI (i) since 1961 till November, 2014.

4. WHO Model List of Essential Medicines 2017.

5. National List of Essential Medicines of India 2011.
6. Kataria BC, Bhavsar VH, Donga BN. Contemplation on approved drugs in India from 1999 through 2011. Asian J Pharm Clin Res. 2012; 5(3):25-29.

7. Goodman and Gilman's. The pharmacological basis of therapeutics. $12^{\text {th }}$ ed. USA: McGraw hill companies; 2008. P. 363.

8. Bertram G. Katzung, Anthony J. Trevor. Basic \& Clinical Pharmacology, 13e. Drugs that act in the central nervous system, Section 5; Introduction to the pharmacology of CNS drugs.

9. Shah S, Patel J, Desai M, Dikshit RK. Critical analysis of antimicrobial and respiratory fixed dose combinations available in Indian market. Int J Med Public Health. 2015; 5(2): 161-64.

10. Pahwa R, Lyons KE, Hauser RA, Fahn S, Jankovic J, Pourcher E, et al. Randomised trial of IPX066, carbidopa/levodopa extended release, in early Parkinson's disease. Parkinsonism and Related Disorders. 2014; 20(2):142-48.

11. Arvind Kumar Yadav, Jitendra Jeenger, Deepika Panwar. Evaluation of rationality of fixed-dose combinations prescribed in psychiatric patients. Natl J Physiol Pharm Pharmacol. 2016; 6(2):150-154. 